\title{
Resection of a giant ovarian tumor the management Challenge
}

\begin{abstract}
A 20year old woman $(100 \mathrm{Kg}, 161 \mathrm{~cm}$ abdominal girth) was admitted for evaluation of massive abdominal distension. Her abdominal ultrasound revealed a giant ovarian tumor. Preoperative interdisciplinary conferences were done in optimizing the patient and planning for the surgery. She was received lying in her heft with difficulty of breathing. Surgical plan at that time was to gradually drain the cyst using local anesthesia under Monitored Anesthesia Care. 15L of cystic fluid was drained preoperatively before the decision to remove the tumor completely under general anesthesia was performed. General anesthesia was induced, with the patient in slight left lateral position with $2 \mathrm{mg}$ Midazolam, $50 \mathrm{mg}$ Ketamine, $3 \mathrm{mg}$ atracurium and $80 \mathrm{mg}$ Succinylcholine. There were episodes of hypotension intraoperatively managed by fluid resuscitation and the use of vasopressors. Intraoperatively, a total of $48 \mathrm{~L}$ of cystic fluid from the mass was removed. Patient was kept intubated and closely monitored post-operatively and was sent home 4 weeks post-surgery improved and stable. This case shows us that it would be safer for a patient with a large ovarian tumor causing cardio respiratory distress to have gradual drainage of cystic fluid first under MAC before considering total removal of the tumor under general anesthesia.
\end{abstract}

Volume 2 Issue 3 - 2015

\author{
Cheryl B Aporado,Vincent Joseph Platon \\ Philippine Society of Anesthesiologists, Philippines
}

Correspondence: Cheryl B Aporado, Jose R Reyes Memoria Medical Center, 2nd floor Anesthesia Office Rizal Avenue Sta, Cruz Manila, Philippines, Tel +639328791318 Email cheryl.010583@gmail.com

Received: March 18, 2015 | Published: May 12, 2015

Keywords: giant ovarian tumor, monitored anesthesia care, general anesthesia

\section{Introduction}

There have been case reports of massive ovarian tumor surgeries until the early 1990's, but recently, there is rarity of cases owing to the improvement and availability of medical and surgical facilities, early detection and management. Large abdominal tumors change the anatomy, physiology and psychology of a patient. Tumors which present themselves within the abdominal cavity may be due to abnormalities in development, inflammatory conditions, cysts, and benign or malignant neoplasm. Whatever the cause, the consequences of surgery are similar and attributable to the size of the tumor rather than to its distinctive pathology. It is with these problems that the choice of anesthesia and management are concerned.

\section{Case Report}

A 20 year old single female was admitted for the first time due to difficulty of breathing and for evaluation of abdominal distention on February 5, 2013. 3years prior to admission, the patient noted a small mass on her lower abdomen with no other associated signs and symptoms. The following year, she noted a gradual increase in the size of her lower abdomen. This increased in size over the next 2 years until the patient became reclusive and ultimately non-ambulatory for 6 months prior to admission. Her family performed her daily hygiene and assisted her changing position several times a day. The patient did not pursue her college education for she was afraid of what society will say regarding her enlarging abdomen.

Physical examination revealed a 20year old woman, lying on her left side. She was alert and oriented but in cardio respiratory distress. Blood Pressure was 90/60 and the heart rate was $110 \mathrm{bpm}$. Her abdomen was noted to be enlarged. The skin overlying the mass shows engorged veins in the anterior chest, abdomen and lower back with an abdominal girth of $161 \mathrm{~cm}$ (Figure $1 \&$ Figure 2). Patient weighed $100 \mathrm{~kg}$. The extremities were without clubbing and cyanosis but there was gross pitting edema of both legs (Figure 3 ). There was ulceration on the left dorsal foot and erythema on left arm. The patient reported having good appetite and regular bowel movement. Her menstrual periods had been regular since age 11 with a duration of 3 days and an interval of 28-30days. Chest x-ray showed poor inspiratory effort secondary to massive abdominal enlargement, as well as, crowding of lung markings due to elevated diaphragm. Whole abdominal ultrasound revealed an extremely large pelvoabdominal unilocular cystic lesion, most likely representing ovarian tumor with mild to moderate post obstructive pyelonephrosis and hydroureter (Figure 4).

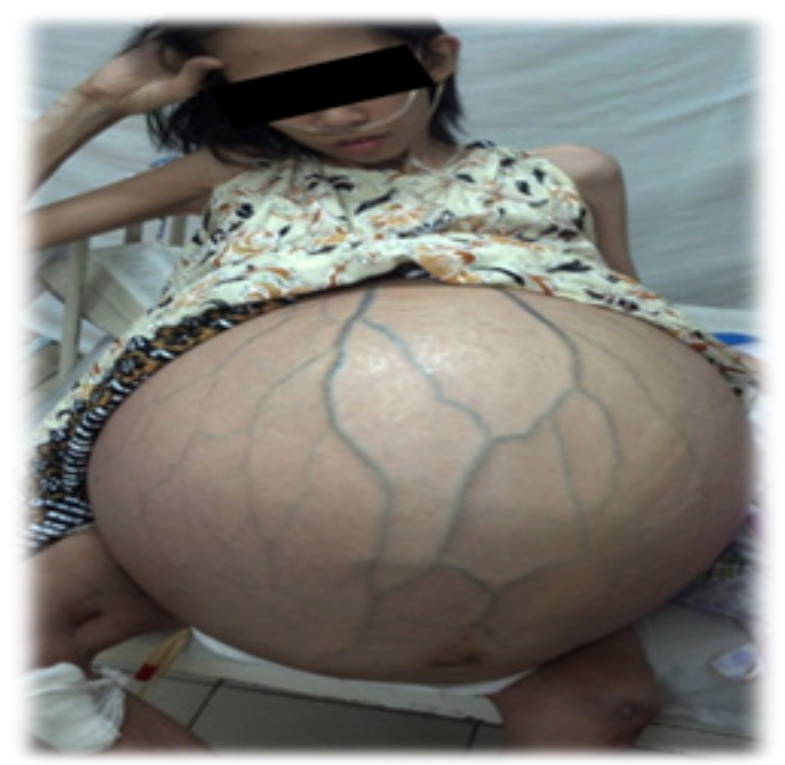

Figure I The skin overlying the mass shows engorged veins in the anterior chest, abdomen and lower back with an abdominal girth of $16 \mathrm{lcm}$. 


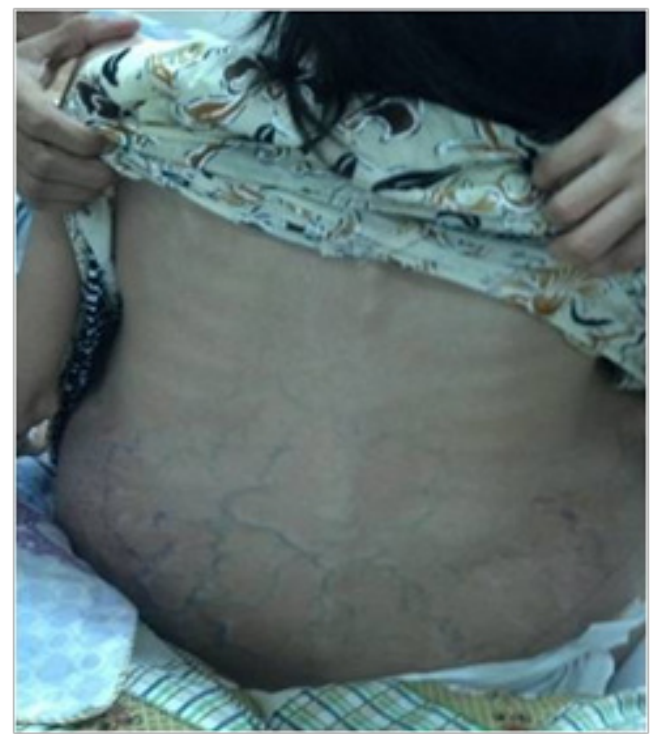

Figure 2 The skin overlying the mass shows engorged veins in the anterior chest, abdomen and lower back with an abdominal girth of $16 \mathrm{Icm}$.

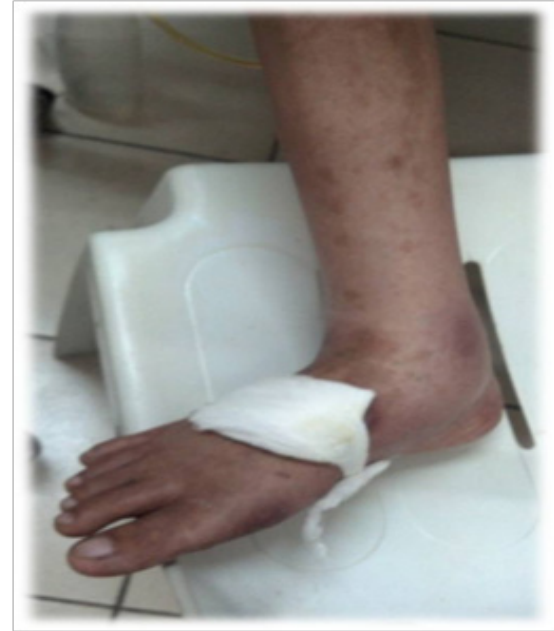

Figure 3 The extremities were without clubbing and cyanosis but there was gross pitting edema of both legs.

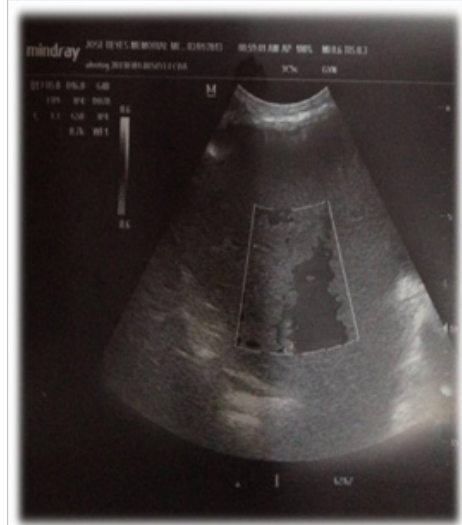

Figure 4 Ovarian tumor with mild to moderate post obstructive pyelonephrosis and hydroureter.

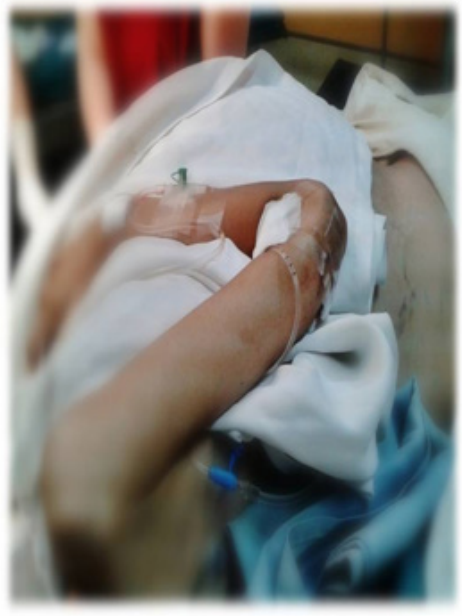

Figure $\mathbf{5}$ Left lateral position to avoid aortocaval compression.

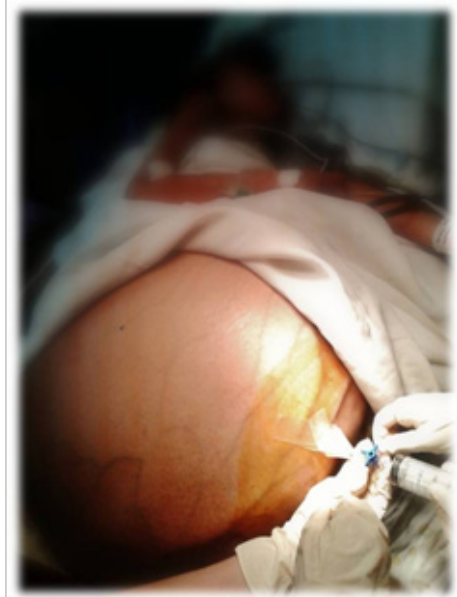

Figure 6 Lidocaine under monitored anesthesia care.

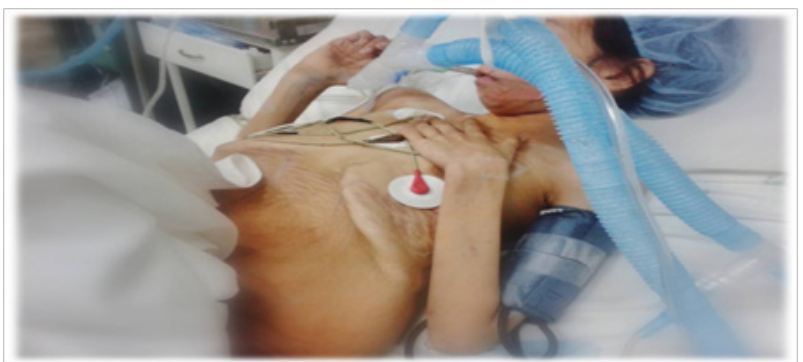

Figure 7 Acquired pneumonia.

ECG revealed Sinus tachycardia with nonspecific ST, T wave changes, low voltage. Hematological data revealed anemia (hemoglobin 84, hematocrit 0.29) hence blood transfusion was done. Biochemical data revealed hypoalbuminemia. Blood gas analysis showed respiratory acidosis. Skin biopsy of ulcer at left foot revealed pyoderma gangrenosum with evidence of keloidal scarring underneath. Skin biopsy of the erythema on left arm was diagnosed to be vasculitis. The patient had acquired pneumonia prior to the day of surgery and was started on antibiotics. An interdisciplinary conferences were held with the Internal Medicine, OB_Gyne, Nutritionist, Dermatology, Anesthesiology and Reconstructive Surgery teams. Patient's 
nutritional status, pulmonary and skin infection were addressed to optimize the patient for the surgery. Members of each team visits the patient daily, and supported the patient in her ultimate decision to have the mass removed.

It was decided to drain the cyst gradually which was to be followed by surgical removal of the mass on the same day. The patient was put on NPO for at least 8 hours before the procedure and Omeprazole $40 \mathrm{mg}$ tab was given at bed time. On the operative day, the patient was taken to the operating room in left lateral position to avoid aortocaval compression (Figure 5) with anti-embolism stockings applied as well. Dopamine infusion was started at $5 \mathrm{ug} / \mathrm{kg}$ and was continued until post surgery. The patient was prepped with betadine and the hospital bed was fully draped with sterile sheets while in lateral position. CVP $\left(7 \mathrm{~cm} \mathrm{H}_{2} \mathrm{O}\right)$ was monitored. Inferior vena cava catheter insertion to monitor CVP was impossible for the large abdomen makes femoral artery and vein identification difficult. Multiparameter monitor which included Arterial BP, HR and percutaneous arterial oxygen saturation was used.

Spontaneous drainage of cyst with echo-guided puncture was done with local anesthesia (Lidocaine) under Monitored Anesthesia Care (Figure 6). This intraoperative drainage of a mass should be reserved for patients with cardiovascular or respiratory compromise due to compression like in this case. ${ }^{1-3} \mathrm{~A}$ total of $15 \mathrm{~L}$ of cystic fluid drained over 4hours with only slight decreases in arterial blood pressure and CVP. Draining was being held when BP decreases, and resumed once managed with fluid and vasopressors. When abdominal distention was noted to be of decreased size. General Endotracheal Anesthesia (Rapid Induction) was then initiated with $3 \mathrm{mg}$ Atracurium, $2 \mathrm{mg}$ Midazolam, 50mg Ketamine, $80 \mathrm{mg}$ Succinylcholine and inhalation of $100 \%$ oxygen. The patient was successfully intubated in the semi left lateral decubitus position. Muscle relaxation was then maintained with atracurium. Intraoperatively, there were some episodes of hypotension managed with volume resuscitation, ongoing dopamine drip titration and vasopressors (ephedrine $5 \mathrm{mg} / \mathrm{ml}$ ).

The surgery lasted for 9hours, wherein adhesions made the resection difficult. Blood loss was $=/>3 \mathrm{~L}$. Cystic fluid drainage was $48 \mathrm{~L}$ and urine output was $2.8 \mathrm{~L}$. Blood transfusion of $1 \mathrm{~L} \mathrm{FWB}$ and 6unit's PRBC, infusion of 1.5L colloid and 4L crystalloids were given. Extubation was delayed to secure the airway. The patient was hooked to mechanical ventilator due to her poor respiratory effort which could also be due to her ongoing hospital acquired pneumonia (Figure 7). She remained in the ICU for another 2 weeks until her lung function and weaning parameters were met. Patient was the subsequently extubated and remained in the hospital for another two weeks. She was discharged well and improved after 4weeks of hospital stay.

\section{Discussion}

The report by Dotters et al. ${ }^{4}$ demonstrates the decreasing incidence of masses this size since the turn of the century and attributes this change to greater health awareness and easier access to medical care. Intraoperative or preoperative drainage of complex masses can result in spillage of tumor cells into the abdominal cavity or drain site with potential for subsequent seeding and can also cause hemorrhage, as well as leaking of cyst fluid into the peritoneal cavity with sudden pulmonary edema as Drife et al. ${ }^{2}$ noted. In previous reports, tumors have been removed, without circulatory or respiratory complications by the gradual drainage of $44-48$. $4 \mathrm{~L}$ of cystic fluid $4-5$ days before the definitive surgery. However, this slow drainage restricts patient's activity and necessitates longer hospital admission. Preoperative or intraoperative drainage of a mass should be reserved for patients with cardiovascular or respiratory compromise due to compression. ${ }^{1-3}$

Dopamine was started for hemodynamic support. Dobutamine was not available though it could be of choice or additional supportive medication for the patient's unstable hemodynamic status. According to the comparative systemic and regional hemodynamic effects of dopamine and dobutamine with cardiomyopathic heart failure by $\mathrm{CV}$ Leier et.al. ${ }^{5}$ During the 24-hr maintenance-dose infusion of each drug, only dobutamine maintained a significant increase of stroke volume, cardiac output, urine flow, urine sodium concentration, creatinine clearance and peripheral blood flow. Drainage of a large simple cyst can be performed preoperatively or intraoperatively, if necessary only after sonographic evidence of mural papillation or element is absent.

In this present case, the sonogram revealed a purely unilocular cystic mass and the patient is in cardio respiratory distress. Hence, a giant ovarian cyst was successfully drained preoperatively and removed on the same day using local anesthesia under Monitored Anesthesia Care followed by General Anesthesia. It was initially planned to do drainage few days before removal of tumor to prevent circulatory depression and reexpansion pulmonary edema. However, the patient could not fully comprehend her medical situation and would have been difficult for her to be restricted for several days more. Therefore, it was decided to drain the cyst and remove the tumor on the same day. Initial cyst drainage required only local anesthesia under MAC. Regional anesthesia was avoided to prevent circulatory depression, vasodilatation and aortocaval compression due to loss of muscle support which could aggravate our patient's cardio-respiratory distress.

General Anesthesia was then decided on for the removal of the tumor due to the following reasons: First, the duration of surgery will be long hence the patient would not be able to tolerate lying still for a long time awake. Second, since the tumor is massive, a large incision was expected. Lastly, to secure the airway for the risk of circulatory depression or re-expansion pulmonary edema after the cyst drainage. The induction of general anesthesia is difficult because of decreased lung and thoracic compliance due to enlarged abdomen. Kobayashi et al. ${ }^{6}$ reported a case of difficult ventilation with premature ventricular contractions. In that case, the trachea was intubated under insufficient depth of anesthesia due to difficult ventilation which induced bronchospasm followed by PVCs.

Patients with large abdominal tumor should be treated as a patient with full stomach wherein rapid sequence induction with cricoid pressure applied. However induction may induce hemodynamic instability when the depth of anesthesia is insufficient and/or anesthesia drugs produce marked cardiovascular depression. This patient received slow intravenous injection of midazolam, ketamine to avoid cardiovascular depression and to add depth of sedation and anesthesia. There was an alteration in BP during change in position from left lateral to supine even with appropriate administration of crystalloid and colloid solutions to keep CVP stable. Pulmonary complications occurred in many patients whose large masses were removed. ${ }^{2,4,7}$ Ulinastatin, a protease inhibitor, is reported to reduce increased pulmonary permeability, ${ }^{8}$ and it might prevent pulmonary edema. ${ }^{9}$ This could have been given to this patient intraoperatively but this is not available in the country.

Respiratory dysfunction can result once the mass is removed due 
to sudden relaxation of the chronically distended, flaccid abdominal and diaphragmatic muscles. ${ }^{10}$ Most notable is a decreased functional residual capacity as a result of an attenuated diaphragm muscle and permanent flaring of the thorax secondary to chronic mass effect on the costochondral junctions. Delayed extubation only after ascertainment of weaning parameters has been recommended ${ }^{4,7}$ as some patients have required reintubation for ventilator failure likely secondary to diaphragm muscle flaccidity. As to this case, the patient did not meet the extubation criteria right after the surgery and her condition was aggravated by pneumonia hence extubation was delayed to secure the airway. Bronchopneumonia has been described in these patients as a result of poor ability to cough and to sustain deep breaths. ${ }^{2}$

\section{Conclusion}

Large intra-abdominal tumors are seen infrequently in modern surgical practice and are often regarded as a challenge. In conclusion, a large ovarian tumor was removed safely by first draining cystic fluid using local anesthesia under MAC to decrease abdominal distention and improve the hemodynamic status of the patient before General Anesthesia with rapid sequence induction was performed.

\section{Recommendations}

The anesthesiologist should be familiar with the alterations in physiology and dilemmas attributed to changes expected with a patient who has a large abdominal mass. We must be aware of possible complications perioperatively and handle them with utmost diligence, knowhow and care. It would have been much better if Dobutamine and/or Norepinephrine was started prior to procedure so as to prevent expected hypotensive and further tachycardic episodes during induction and during the procedure itself.

\section{Acknowledgements}

None.

\section{Conflict of interest}

The author declares no conflict of interest.

\section{References}

1. Beacham WD, Webster HD, Lawson EH, et al. Uterine and/or ovarian tumors weighing 25 pounds or more. Am J Obstet Gynecol. 1971;109(8):1153-1161.

2. Drife JO, Trotter GA. Britain's biggest ovarian cyst? Br Med J (Clin Res Ed). 1981;283(6307):1661-1662.

3. Pretorius RG, Malory WE, La Fontaire D. Management of massive ovarian tumors. Surg Gynecol Obstet. 1989;169(6):532-536.

4. Dotters DJ, Katz VL, Curie J. Massive ovarian cyst: A comprehensive surgical approach. Obstet Gynecol Surv. 1988;43(4):191-196.

5. Leier CV, Heban PT, Huss P, et al. Comparative systemic and regional hemodynamic effects of dopamine and dobutamine in patients with cardiomyopathic heart failure. Circulation. 1978;58(3 Pt 1):466-475.

6. Kobayashi T, Yokoyama k. Anesthetic management of a patient with a giant ovarian tumor complicated with cerebral palsy. Masui. 1990;39(9):1234 1238.

7. Matory W, Pretorius RG, Hunter RE, et al. A new approach to massive abdominal tumors using immediate abdominal wall reconstruction. Plast Reconstr Surg. 1989;84(3):442-448.

8. Arakawa M, Kambara k, Miyazaki H, et al. Effects of Ulinastatin, an antiprotease, on alloxan-induced lung injury in dogs. Clin Ther. 1992;14(3):396-408.

9. Tanaka k, Nozaki S, Hayashi k, et al. Re-expansion pulmonary edema after removal of a giant mediastinal tumor. (Japanese) 5 Clin Anesth. 1994;18:1459-1460.

10. Hunter DJ. Management of a massive ovarian cyst. Obstet Gynecol. 1980;56(2):254-255. 